\title{
Vibration-rotation spectra of hydrogen halides in rare-gas liquids: Q-branch absorption*
}

\author{
A. Medina ${ }^{1, \ddagger}$, J. M. M. Roco², A. Calvo Hernández², and S. Velasco ${ }^{2}$ \\ ${ }^{1}$ ETSII de Béjar, Universidad de Salamanca, 37700, Béjar, Salamanca, Spain; \\ ${ }^{2}$ Departamento de Física Aplicada, Facultad de Ciencias, Universidad de \\ Salamanca, 37008 Salamanca, Spain
}

\begin{abstract}
Near-infrared spectra of $\mathrm{HCl}$ highly diluted in liquid $\mathrm{Ar}$ show intense absorption in the P-R interbranch region, so-called Q-branch absorption. In spite of its relevance for the shape of the bands, its physical origin has been elusive to date. We employ molecular dynamics simulations to study the influence of some physical effects that could contribute to Q-branch absorption. We check that multipole-induced dipole induction mechanisms are not quantitatively relevant in this spectral region. We show that the particular characteristics of accurate $\mathrm{HCl}-\mathrm{Ar}$ anisotropic potentials and the peculiar hindered rotational motion they provoke on the diatomic probe are essential to understand Q-branch absorption.
\end{abstract}

\section{INTRODUCTION}

The fundamental vibration-rotation absorption bands of some hydrogen halides ( $\mathrm{HF}, \mathrm{HCl}$ ) dissolved in condensed rare-gas or spherical $\left(\mathrm{SF}_{6}, \mathrm{CCl}_{4}\right)$ solvents show, apart from the well-known $\mathrm{P}$ - and R-branches, a third branch located in the spectral region between the former. This is called the Q-branch, and it is dipole-forbidden for isolated heteronuclear diatomics. Its relative intensity grows with decreasing rotational constant of the diatomic, increasing density and decreasing temperature [1]. In some particular systems ( $\mathrm{HCl}-\mathrm{Ar}$ doped with $\mathrm{Kr}$ or $\mathrm{Xe}, \mathrm{HCl}-\mathrm{CCl}_{4}$ ), the Q-branch dominates the observed spectral profiles. Its first observation is dated around 1958 by Bulanin et al. [2] and Lascombe et al. [3], but no consensus has emerged to date on a clear physical interpretation of absorption in that spectral region.

Although theoretical attempts have been developed [4], the analysis of experimental results is still the main approach to the physical mechanisms at the origin of Q-branch absorption. The existence of some kind of rotational hindrance in the motion of the solute is supported by the work of Chesnoy [5] and Rutkowski et al. [6], in which it is shown that in the evolution from liquid to solid solvents the central component of the spectrum disappears. Underlying this observation is the role of the symmetry of the nearest environment around the probe. Another hypothesis is the possible influence of interactioninduced contributions to the dipole moment of the solution that could become relevant in the spectral region of the Q-branch. This is supported by the weakening of Q-branch absorption when measuring the first overtone band, indicating that some kind of frequency-dependent mechanism should be present [7].

Related to the eventual hindrance of rotational motion, there is a point that makes these studies especially attractive: the possible existence of weakly bound solute-solvent complexes in liquids and their nature. In this respect, Tokhadze et al. [8] measured with high resolutions the absorption spectra

\footnotetext{
*Lecture presented at the European Molecular Liquids Group (EMLG) Annual Meeting on the Physical Chemistry of Liquids: Novel Approaches to the Structure, Dynamics of Liquids: Experiments, Theories, and Simulation, Rhodes, Greece, 7-15 September 2002. Other presentations are published in this issue, pp. 1-261.

ॠCorresponding author
} 
of the most intense librational mode of HF...B (with $\mathrm{B}=\mathrm{Ar}, \mathrm{Xe}, \mathrm{CO}, \mathrm{CO}_{2}$ ) from the gas phase to the liquid. They concluded that the observed Q-branch in the liquid is the resulting enhancement of such librational mode, and thus, propose that Q-branches reveal the coexistence of free and complexed molecules in the liquid.

Our objective in this paper is double, first we shall quantify the importance of electric multipolar interaction-induced effects in the region between $\mathrm{P}$ - and R-branches for $\mathrm{HCl}$ highly diluted in liquid Ar. Second, we are interested in the eventual relationship between solute-solvent anisotropic interactions and the rotational hindrance of the molecular probe. By means of molecular dynamics (MD) simulations we test a semiclassical model that reproduces the third absorption peak in the spectra without the assumption of any adjustable parameter.

\section{EVALUATION OF INTERACTION-INDUCED EFFECTS}

We calculate the near-infrared absorption coefficient of a diluted solution of diatomic molecules in a nonpolar solvent as given by linear response theory:

$$
\alpha(\omega) \simeq \omega \sinh \left(\frac{\beta \hbar \omega}{2}\right) I(\omega)
$$

with

$$
I(\omega)=\int_{0}^{\infty} \cos (\omega t) C_{R V}(t) d t
$$

where $C_{R V}(t)$ is the time-correlation function (TCF) associated to the optically active degrees of freedom in the near-infrared region. The frequency distribution of intensity is accounted for by a semiclassical population factor [9] arising from detailed balance condition and the classical correspondence limit. In view of the large differences between the time scales of vibration $(V)$ and rotation $(R)$ of the diatomic, it is possible to approximate $C_{R V}(t)$ as:

$$
C_{R V}(t) \simeq C_{V}(t) C_{R}(t)=<\Delta x(0) \Delta x(t)><\boldsymbol{M}(0) \cdot \boldsymbol{M}(t)>
$$

where $\Delta x(t)$ represents the separation of the instantaneous internuclear distance, $x(t)$, from its equilibrium value, $x_{e}$, and $\boldsymbol{M}(t)$ is the total dipole moment of the mixture. We shall consider contributions to $\boldsymbol{M}(t)$ both from the permanent dipole moment of the diatomic, $\boldsymbol{\mu}_{0}(t)$, and also from the dipole moments induced in the solvent atoms by the total electric field, $\boldsymbol{E}_{j}(t)$, originating from the multipole moments of the diatomic, $Q_{j}(t)$. Thus, the relevant orientational TCF, $C_{R}(t)$, to obtain the $(1 \leftarrow 0)$ fundamental vibration-rotation band is

$$
\begin{aligned}
C_{R}(t) \sim & \mu_{0}^{\prime 2}\langle\boldsymbol{\mu}(0) \cdot \boldsymbol{\mu}(t)\rangle+\alpha^{2} \sum_{j=0}^{k} Q_{j}^{\prime 2}\left\langle\boldsymbol{E}_{j}(0) \cdot \boldsymbol{E}_{j}(t)\right\rangle \\
& +2 \alpha \mu_{0}^{\prime} \sum_{j=0}^{k} Q_{j}^{\prime}\left\langle\boldsymbol{\mu}(0) \cdot \boldsymbol{E}_{j}(t)\right\rangle+2 \alpha^{2} \sum_{i, j=0}^{k} Q^{\prime} Q_{j}^{\prime}\left\langle\boldsymbol{E}_{i}(t) \cdot \boldsymbol{E}_{j}(t)\right\rangle
\end{aligned}
$$

where $\boldsymbol{\mu}(t)$ is a unit vector along the axis of the diatomic and $\alpha$ the isotropic polarizability of the solvent. The parameters that give the relative intensities of the different contributions are the bond-length derivatives of the multipole moments of the molecule: for $\left.j=0, Q_{0}^{\prime} \equiv \mu_{0}^{\prime}=\partial \mu_{0} / \partial x\right)_{x_{e}}$ and for $j>0, Q_{j}^{\prime} \equiv$ $\left.\partial Q_{j} / \partial x\right)_{x_{e}}$. These data, although scarce, are available in the literature. From simulations we calculate $C_{V}(t)$ and $C_{R}(t)$, and by performing the corresponding Fourier transforms (eqs. 1,2), the contributions to $\alpha(\omega)$ are obtained:

$$
\alpha(\omega)=\alpha_{\mathrm{P}}(\omega)+\alpha_{\mathrm{I}}(\omega)+\alpha_{\mathrm{PI}}(\omega)+\alpha_{\mathrm{II}}(\omega)
$$


where the subscripts denote permanent $(\mathrm{P})$, pure induced (I), cross-terms between the permanent and induced contributions (PI), and cross-terms between different induced contributions (II). Each addend is directly associated to the corresponding TCF in eq. 4.

In the simulations, we consider a diluted solution formed by one diatomic molecule and 250 solvent atoms. The internal vibrational motion of the diatomic is modeled through a Morse potential and solute-solvent interactions through standard atom-atom potentials [10] (applying Lorentz-Berthelot combination rules when necessary). We shall detail later the question of solute-solvent anisotropic interaction potentials.

We have calculated $\alpha(\omega)$ for $\mathrm{HCl}$ in liquid $\mathrm{Ar}$ at $T=115 \mathrm{~K}$, where recent experiments [1] corroborate the existence of important absorption contributions in the P-R interbranch region (see the peak around $2875 \mathrm{~cm}^{-1}$ in Fig. 1). We computed induced contributions up to $k=2$, i.e., dipole-induced dipole, quadrupole-induced dipole, and octupole-induced dipole terms, taking the bond-length derivatives $Q_{i}^{\prime}$ from the literature [11]. We did check that certain induced contributions always display a maximum in the P-R interbranch region, but their intensities are quantitatively small compared to the permanent contribution. Defining the relative intensities as the maximum height of the different terms of eq. 5 with respect to the permanent one, we find that:

$$
\frac{\alpha_{\mathrm{I}, \max }}{\alpha_{\mathrm{P}, \max }}=1.4 \times 10^{-2} ; \quad \frac{\alpha_{\mathrm{PI}, \max }}{\alpha_{\mathrm{P}, \max }}=-5.0 \times 10^{-2} ; \quad \frac{\alpha_{\mathrm{I}, \max }}{\alpha_{\mathrm{P}, \max }}=1.7 \times 10^{-3}
$$

These numbers show that induction mechanisms amount, at best, to $1.4 \%$ of the permanent one. Moreover, there are at least partial cancellation effects since cross permanent-induced terms present a negative maximum. So, it seems that this kind of induced contributions (in principle, the largest we could expect, disregarding anisotropic polarizability, hyperpolarizability, or back-induction) do not represent the basic physical mechanism responsible for Q-branch absorption. These results are in accordance with those of Chatzis and Samios [12] who recently showed that for $\mathrm{HCl}$ dissolved in a highly polarizable solvent such as $\mathrm{CCl}_{4}$, contributions from dipole-induced dipole and back-induction mechanisms are small.

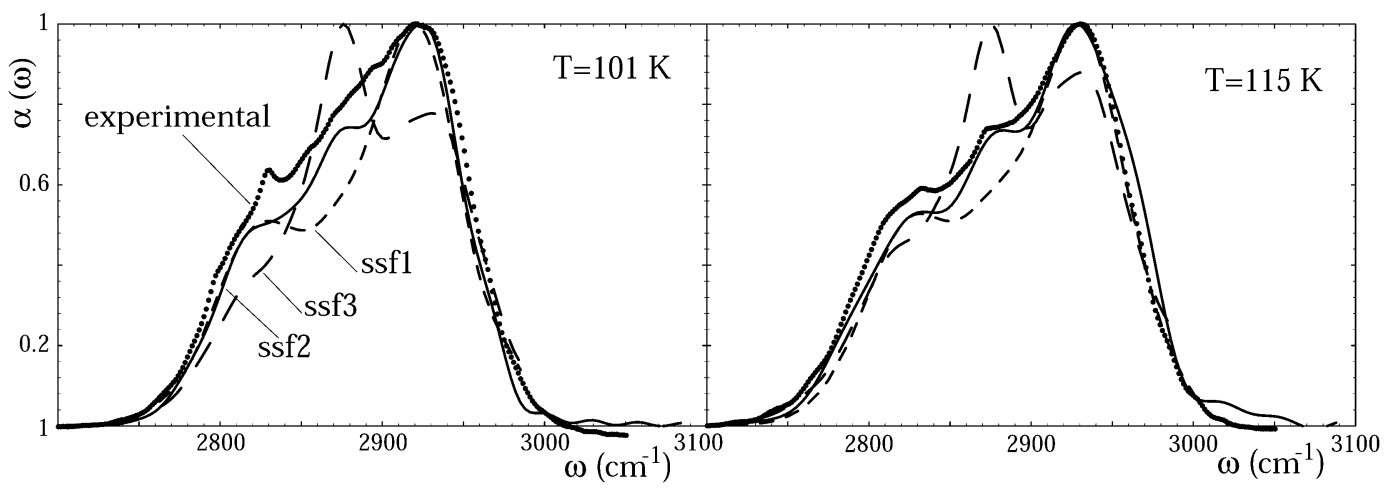

Fig. 1 Experimental and simulated infrared fundamental band of $\mathrm{HCl}$ in liquid $\mathrm{Ar}$.

\section{HCI-Ar ANISOTROPIC INTERACTIONS AND ROTATIONAL HINDRANCE}

One of our aims is to study the sensitivity of the shape of the near-infrared spectral profiles with respect to solute-solvent anisotropic interactions. Previous MD results obtained with standard site-site potentials built up from atom-atom 6-12 Lennard-Jones potentials did not satisfactorily reproduce the absorption in the P-R interbranch region [10]. We shall make use here of three anisotropic $\mathrm{HCl}-\mathrm{Ar}$ interaction potentials originally obtained as site-site fits to three realistic energy surfaces, HWK I and 
HWK II by Holmgren et al. [13] and M5 by Hutson et al. [14]. We are not especially interested in the quality of the fits, so we consider these site-site anisotropic potentials as prototypical effective surfaces. From the comparison of the diverse spectral profiles that originate from the simulations, we can learn about the physical origin of Q-branch absorption. We shall denote our site-site fitted (ssf) potentials as ssf1 (fit to HWK I), ssf2 (fit to HWK II), and ssf3 (fit to M5).

All these ssf surfaces incorporate a basic characteristic shown by any realistic interaction potential: an absolute minimum at linear $\mathrm{Ar}-\mathrm{H}-\mathrm{Cl}$ orientation. Eventually, this reproduces the existence of linear weakly bound van der Waals complexes at gas densities. The parameters of the potentials are compiled in Table 1.

Table 1 Lennard-Jones parameters of the considered $\mathrm{HCl}-\mathrm{Ar}$ anisotropic interaction surfaces.

\begin{tabular}{lrrrrrr}
\hline & \multicolumn{3}{c}{$\varepsilon(\mathrm{K})$} & & \multicolumn{3}{c}{$\sigma(\AA)$} \\
\cline { 2 - 7 } & \multicolumn{1}{c}{ ssf1 } & \multicolumn{1}{c}{ ssf2 } & \multicolumn{1}{c}{ ssf3 } & ssf1 & ssf2 & ssf3 \\
\hline $\mathrm{H}-\mathrm{Ar}$ & 77.5 & 79.5 & 120.0 & 2.36 & 2.41 & 2.46 \\
$\mathrm{Cl}-\mathrm{Ar}$ & 222.0 & 150.3 & 147.8 & 3.43 & 3.44 & 3.42 \\
\hline
\end{tabular}

The experimental vibration-rotation fundamental bands of $\mathrm{HCl}$ in liquid $\mathrm{Ar}$ at two different temperatures along the gas-liquid coexistence curve [1] are displayed in Fig. 1. Both spectra lack fine structure and are thus susceptible of quasi-classical MD studies. At the highest temperature ( $T=115 \mathrm{~K})$, the band displays an apparent absorption peak corresponding to Q-branch absorption.

At $T=101 \mathrm{~K}$, although Q-branch absorption does not provoke a visible peak, the P-R interbranch region has a peculiar quasilinear behavior, indicative of the existence of this specific absorption. Whenever this absorption is absent, the spectra show a deep valley between P- and R-branches.

The same figure also includes the simulated spectra obtained with the mentioned ssf potentials. All of them allow us to reproduce the width of the band with small discrepancies with respect to experiments (within the boundaries one can expect as reasonable from semiclassical calculations). Model ssf1 allows a fair estimation of the width of the R-branch but underestimates the relative intensity of the P-branch. Its main failure appears in the P-R interbranch region where it predicts an absorption shallow valley without apparent Q-branch. In the spectra obtained with potential ssf2 the Q-branch is visible. For $T=115 \mathrm{~K}$, in spite of a slight underestimation of the relative intensity of the P-branch, the comparison between simulations and experiment is noticeably good. For the lowest temperature, the absorption intensity is lower than the experimental one, but qualitatively the relative intensities of the maxima giving the structure of the profile are fairly well reproduced. Nevertheless, the absorption band obtained from ssf3 differs in an important manner from experiments. At both conditions the simulations predict a large Q-branch peak that gathers absorption intensity from P- and R-branches (respectively to ssf2 the Q-branch intensity is enlarged by about $25 \%$ ). The global width of the band is also well reproduced, but its structure is far from being as experiments show. In conclusion, the absorption profile, particularly in the P-R interbranch region, appears to be interestingly sensitive to the particular shape of ssf potentials.

The permanent dipole moment TCF, $C_{P}(t)$, reflect the differences among different anisotropic potentials. In Fig. 2, we depict $C_{P}(t)$ as calculated from MD simulations $\left[C_{P}^{M D}(t)\right]$ for the ssf potentials considered. For the sake of comparison, the corresponding theoretical function, $C_{P}^{t h}(t)$, obtained within the classical rigid rotor model for each temperature [15], is also represented. The comparison of any of the simulated functions with $C_{P}^{t h}(t)$ leads us to conclude that: (a) the short time behavior of $C_{P}^{M D}(t)$ and $C_{P}^{t h}(t)$ is similar, although slightly slower for $C_{P}^{M D}(t)$; (b) for any of the checked ssf potentials, $C_{P}^{M D}(t)$ presents an evident damped oscillatory behavior at intermediate times $(0.2-0.8 \mathrm{ps})$; and (c) $C_{P}^{\text {th }}(t)$ reaches the equilibrium through a monotonic positive slope with negative values, but $C_{P}^{M D}(t)$ oscillates 


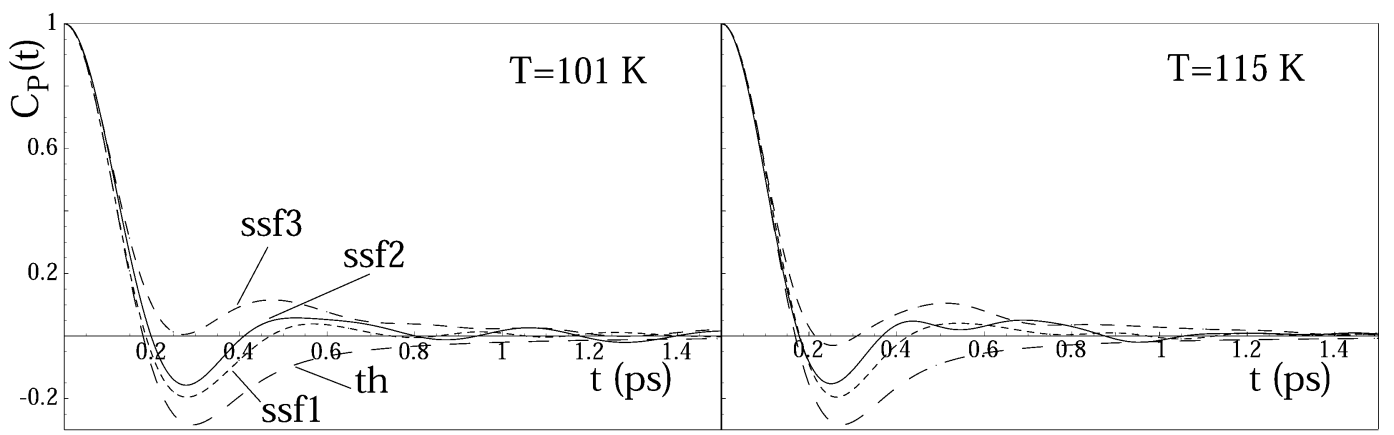

Fig. 2 Simulated permanent dipole moment TCF obtained with different ssf potentials.

around the equilibrium value with changing sign and decreasing amplitude. The time at which all functions reach the equilibrium zero value is similar, around $1.5 \mathrm{ps}$. The abrupt increase of Q-branch absorption in the spectra evolves in the order ssf1-ssf2-ssf3 and the character of the motion evolves toward a more hindered rotation in the same order.

\section{SUMMARY AND CONCLUSIONS}

The experimental near-infrared spectra of $\mathrm{HCl}$ in liquid Ar over the gas-liquid coexistence line present intense absorption in the spectral region between R- and P-branches, the so-called Q-branch. Its physical interpretation is not clear up to date. One of the several theoretical ideas proposed to explain this particular absorption is the existence of electric-induced contributions to the dipole moment of the solution. By means of MD simulations, we have checked that at least multipole-induced dipole contributions are not quantitatively relevant in this spectral region. Other possible explanations suggested essentially by experimental works relate Q-branch absorption with the anisotropic environment close to the diatomic. We have shown that for $\mathrm{HCl}$ in liquid $\mathrm{Ar}$, the experimentally observed intensity of Q-branch could be reproduced by means of site-site fittings to realistic $\mathrm{HCl}-\mathrm{Ar}$ anisotropic potentials incorporating an absolute minimum at $\mathrm{Ar}-\mathrm{H}-\mathrm{Cl}$ orientations. This results in a peculiar hindering in the rotational motion of the diatomic probe. A challenging future work would consist in exploring the eventual relationship between Q-branch absorption and the existence of some kind of $\mathrm{Ar}{ }^{\cdots} \mathrm{HCl}$ complexes in the liquid phase.

\section{ACKNOWLEDGMENTS}

We acknowledge financial support from the Ministerio de Educacin y Ciencia of Spain (Grant No. BFM2002-01225/FEDER) and from JCyL (Grant No. SA097/01).

\section{REFERENCES}

1. A. Medina, J. M. M. Roco, A. Calvo Hernández, S. Velasco, M. O. Bulanin, W. A. Herrebout, B. J. van der Veken. J. Chem. Phys. 116, 5058 (2002); A. Medina, J. M. M. Roco, A. Calvo Hernández, S. Velasco. J. Chem. Phys. 119, 5176 (2003).

2. M. O. Bulanin and N. D. Orlova. Opt. Spectrosc. 4, 569 (1958).

3. J. Lascombe, P. V. Huong, M. L. Josien. Bull. Soc. Chim. France 1175 (1959).

4. See refs. [14] and [15] in paper [1].

5. J. Chesnoy. Chem. Phys. Lett. 114, 220 (1985).

6. K. S. Rutkowski and S. M. Melikova. J. Mol. Struct. 448, 231 (1998). 
7. M. O. Bulanin, S. Velasco, A. Calvo Hernández. J. Mol. Liq. 70, 107 (1996).

8. K. G. Tokhadze and Z. Mielke. J. Chem. Phys. 99, 5071 (1993); K. G. Tokhadze, S. S. Utkina, N. N. Filippov, Z. Mielke. Opt. Spectrosc. 79, 536 (1995).

9. P. H. Berens and K. R. Wilson. J. Chem. Phys. 74, 4872 (1981).

10. J. A. Padró and E. Guàrdia. J. Mol. Liq. 70, 199 (1996).

11. H. Hettema, P. S. Wormer, A. J. Thakkar. Mol. Phys. 80, 533 (1993).

12. G. Chatzis and J. Samios. J. Phys. Chem. A 105, 9522 (2001).

13. S. L. Holmgren, M. Waldman, W. Klemperer. J. Chem. Phys. 69, 1661 (1978).

14. J. M. Hutson and B. J. Howard. Mol. Phys. 45, 769 (1982).

15. J.-P. Hansen and I. R. McDonald. Theory of Simple Liquids, p. 497, Academic Press, London (1991) 